\title{
Damping of kink waves by mode coupling
}

\section{Analytical treatment ${ }^{\star}$}

\author{
A. W. Hood ${ }^{1}$, M. Ruderman ${ }^{2}$, D. J. Pascoe ${ }^{1}$, I. De Moortel ${ }^{1}$, J. Terradas ${ }^{3}$, and A. N. Wright ${ }^{1}$ \\ ${ }^{1}$ School of Mathematics and Statistics, University of St Andrews, St Andrews, KY16 9SS, UK \\ 2 Solar Physics and Space Plasma Research Centre (SP2RC), University of Sheffield, Sheffield, S3 7RH, UK \\ ${ }^{3}$ Departament de Física, Universitat de les Illes Balears, 07122 Palma de Mallorca, Spain \\ e-mail: alan@mcs.st-andrews.ac.uk
}

Received 24 October 2012 / Accepted 2 January 2013

\section{ABSTRACT}

\begin{abstract}
Aims. We investigate the spatial damping of propagating kink waves in an inhomogeneous plasma. In the limit of a thin tube surrounded by a thin transition layer, an analytical formulation for kink waves driven in from the bottom boundary of the corona is presented.

Methods. The spatial form for the damping of the kink mode was investigated using various analytical approximations. When the density ratio between the internal density and the external density is not too large, a simple differential-integral equation was used. Approximate analytical solutions to this equation are presented.

Results. For the first time, the form of the spatial damping of the kink mode is shown analytically to be Gaussian in nature near the driven boundary. For several wavelengths, the amplitude of the kink mode is proportional to $\left(1+\exp \left(-z^{2} / L_{\mathrm{g}}^{2}\right)\right) / 2$, where $L_{\mathrm{g}}^{2}=16 / \epsilon \kappa^{2} k^{2}$. Although the actual value of 16 in $L_{\mathrm{g}}$ depends on the particular form of the driver, this form is very general and its dependence on the other parameters does not change. For large distances, the damping profile appears to be roughly linear exponential decay. This is shown analytically by a series expansion when the inhomogeneous layer width is small enough.
\end{abstract}

Key words. magnetohydrodynamics (MHD) - Sun: atmosphere - Sun: corona - Sun: magnetic topology - Sun: oscillations - waves

\section{Introduction}

Coronal multi-channel polarimeter (CoMP) observations have revealed periodic Doppler shift oscillations propagating along large, off-limb coronal loops (Tomczyk et al. 2007; Tomczyk $\&$ McIntosh 2009). From the analysis of the ratio of outward and inward propagating power along loop structures, Tomczyk $\&$ McIntosh (2009) found a strong decay in the wave amplitudes as they travelled along the loop. Indeed, only shorter loops show evidence of inward power, implying the presence of either very efficient dissipation or mode conversion. Furthermore, McIntosh et al. (2011) demonstrated that these propagating, transverse loop displacements carry a significant amount of energy and hence could potentially play an important role in coronal heating (Parnell \& De Moortel 2012) and/or the solar wind acceleration (Ofman 2010). Finally, the ubiquitous nature of these waves makes them very attractive as a potential seismological tool (De Moortel \& Nakariakov 2012). Similar transverse oscillations have also been reported in spicules (De Pontieu et al. 2007; He et al. 2009), X-ray jets (Cirtain et al. 2007) and prominence fibrils (Okamoto et al. 2007).

The observed waves show clear, periodic variations in Doppler shifts (velocities) but only very weak signatures in intensity. This incompressible nature, together with the fact that the observed speeds are generally of the order of the local Alfvén speed gives the waves a distinct Alfvénic character (Goossens et al. 2009). Motivated by the numerous observations of waves in coronal loops, for example in Tomczyk et al. (2007), we recently performed a series of 3D numerical

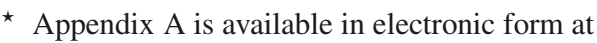
http://www. aanda.org
}

simulations (Pascoe et al. 2010, 2011, 2012). Using 3D simulations of loop displacements, Pascoe et al. (2010) clarified the nature of the observed loop displacements as coupled kink-Alfvén waves: transverse footpoint motions travel along the loop and through the inhomogeneity at the loop boundary, couple efficiently to (azimuthal) Alfvén waves. This mode coupling takes place at locations where phase speed of the propagating kink mode matches the local Alfvén speed (Allan \& Wright 2000) and energy is transferred from the transverse kink modes to the Alfvén modes in the shell regions of the loop. Hence, the kink modes are effectively a moving source of Alfvén waves until all energy is transferred into the Alfvén waves. Pascoe et al. (2010) showed that this coupling is sufficiently efficient to qualitatively explain the observed rapid amplitude decay. This mode coupling takes place even for modest density contrasts or an arbitrary inhomogeneous medium (Pascoe et al. 2011). Further evidence for the occurence of the mode coupling presence can be found in the frequency filtering which is inherent to this mechanism. Indeed, Terradas et al. (2010) demonstrated that the damping of the transverse motions through mode coupling is frequencydependent, with higher frequencies leading to shorter damping lengths. Subsequently, Verth et al. (2010) found evidence for this frequency in the CoMP data of Tomczyk \& McIntosh (2009), strengthening the interpretation of the observed, propagating Doppler shift oscillations as coupled kink-Alfvén waves. Additional effects such as the presence of a background flow or gravitational stratification on the mode coupling mechanism were studied by Soler et al. (2011a,b).

The damping lengths obtained through mode coupling do not only depend on the frequency of the footpoint displacements but also on the width of the inhomogeneous layer at the edge 
of the loop. Wider layers lead to more efficient mode coupling and, hence, shorter damping lengths. The periods observed by Tomczyk et al. (2007) are of the order of several minutes (peaking at about $5 \mathrm{~min}$ ) and to achieve the observed strong damping in this period range, a relatively wide layer, of the order of half of the loop radius, is required (Pascoe et al. 2010, 2012). Similar evidence for loops with wide boundary layers was obtained by seismological estimates derived from the damping rate of standing, kink mode oscillations by Goossens et al. (2002). Similar to the mode coupling mechanism described by Pascoe and coauthors, which operated on open flux tubes, standing, kink mode oscillations, on closed field lines, also undergo efficient damping through resonant absorption in the presence of an inhomogeneous shell region (e.g. Hollweg \& Yang 1988; Goossens et al. 1992; Ruderman \& Roberts 2002).

The numerical simulations of Pascoe et al. (2012) revealed a change in the characteristic damping profile of the transverse velocity displacements: at low distance, a Gaussian profile of the form $\exp \left(-z^{2}\right)$ is present, followed by an exponential damping profile of the form $\exp (-z)$ at larger distances. The distance at which this transition between the two damping profiles occurs appears to depend on the density profile. For narrow inhomogeneous layers, the exponential damping profile is evident after only a few wavelengths. However, for wider shell regions, the damping profile is largely Gaussian except at large distances. As observational evidence (both for propagating and standing kink mode oscillations) suggests the presence of wide inhomogeneous shell regions, Pascoe et al. (2012) suggested the Gaussian profile might be best suited to the observed CoMP data. A transition to the exponential damping regime would still occur at larger distances but, due to the strong damping, the amplitudes of the Doppler shift oscillations are expected to be too small to be observationally relevant.

A related study for the time dependent evolution of a standing kink mode has been undertaken by Ruderman \& Terradas (2012). They find that the temporal behaviour of the kink mode amplitude is damped in a Gaussian manner for small times, before approaching a linear exponential damping for large times.

In this paper, we investigate the mode coupling process analytically to clarify the nature of the Gaussian damping profile. The paper is organised as follows. The analytical problem is formulated in Sect. 2, followed by the derivation of the governing equations in Sect. 3. The nature of the damping profile is investigated in Sect. 4 by using various approximations. The key point of this section is to demonstrate when previously used approximations are valid and when they fail. These approximations are compared with numerical simulations in Sect. 5. Conclusions are presented in Sect. 6.

\section{Problem formulation}

We consider the propagation of kink waves in a semi-infinite magnetic tube. In cylindrical coordinates $r, \varphi, z$ with the $z$-axis coinciding with the tube axis, the tube is situated in the halfspace $z>0$. The equilibrium magnetic field is in the $z$-direction, $\boldsymbol{B}_{0}=\left(0,0, B_{0}\right)$, and homogeneous. The equilibrium density, $\rho(r)$ is given by

$\rho(r)=\left\{\begin{array}{cc}\rho_{\mathrm{i}}, & r \leq R-\ell / 2, \\ \rho_{t}(r), & R-\ell / 2 \leq r \leq R+\ell / 2, \\ \rho_{\mathrm{e}}, & r \geq R+\ell / 2,\end{array}\right.$

where $\rho_{\mathrm{i}}$ and $\rho_{\mathrm{e}}$ are constants, and $\rho_{\mathrm{i}}>\rho_{\mathrm{e}}$. Thus, the Alfvén speed, $V_{\mathrm{A}}(r)=B_{0} / \sqrt{\mu_{0} \rho(r)}$, is a function of radius alone and we use the notation

$V_{\mathrm{A}}(r)=\left\{\begin{array}{cc}V_{\mathrm{i}}, & r \leq R-\ell / 2, \\ V_{\mathrm{A}}(r), & R-\ell / 2 \leq r \leq R+\ell / 2, \\ V_{\mathrm{e}}, & r \geq R+\ell / 2 .\end{array}\right.$

The tube is excited by imposing a velocity perturbation at $z=0$. Thus, waves are excited and these propagate into the system, namely $z>0$. We select the external Alfvén speed to be $1 \mathrm{Mm} \mathrm{s}^{-1}$. Distances are measured in $\mathrm{mm}$ and periods in seconds.

To demonstrate the Gaussian nature of the damping analytically, we neglect stratification, field line curvature and expansion of the magnetic tube. The importance of these physical phenomena will be discussed in Sect. 6 but we expect them to be unimportant when the wavelength and damping lengths are shorter than the gravitational scale height, the radius of curvature and the characteristic spatial scale of the tube's radial variation. These conditions may not necessarily be met in all coronal flux tubes but are imposed for mathematical simplicity.

In this paper, we are interested in linearly polarized (transverse) oscillations. The displacements in the radial and azimuthal directions are taken as $\xi_{r}=\xi_{r}(r, z, t) \cos \varphi$ and $\xi_{\varphi}=$ $-\xi_{\varphi}(r, z, t) \sin \varphi$, respectively, and the perturbed total pressure as $P=B_{0} b_{z} / \mu_{0}=P(r, z, t) \cos \varphi$, where $b_{z}$ is the $z$-component of the magnetic field perturbation. This is a lateral kink mode (as opposed to the helical kink) and, due to form of the equilibrium, there is no coupling to other azimuthal mode numbers.

The linearised Magnetohydrodynamic (MHD) equations, describing the plasma evolution in the cold plasma limit, are given in Ruderman (2011, hereafter Paper I) and they reduce to

$$
\begin{aligned}
& \frac{\partial\left(r \xi_{r}\right)}{\partial r}-\xi_{\varphi}=0, \\
& \mathcal{L} \xi_{r} \equiv \frac{\partial^{2} \xi_{r}}{\partial z^{2}}-\frac{1}{V_{\mathrm{A}}^{2}} \frac{\partial^{2} \xi_{r}}{\partial t^{2}}=\frac{\mu_{0}}{B_{0}^{2}} \frac{\partial P}{\partial r}, \\
& \mathcal{L} \xi_{\varphi} \equiv \frac{\partial^{2} \xi_{\varphi}}{\partial z^{2}}-\frac{1}{V_{\mathrm{A}}^{2}} \frac{\partial^{2} \xi_{\varphi}}{\partial t^{2}}=\frac{\mu_{0}}{B_{0}^{2}} \frac{P}{r}
\end{aligned}
$$

For notational simplicity, we define the differential operators

$$
\begin{array}{ll}
\mathcal{L}_{\mathrm{k}}=\frac{\partial^{2}}{\partial z^{2}}-\frac{1}{C_{\mathrm{k}}^{2}} \frac{\partial^{2}}{\partial t^{2}}, & \mathcal{L}=\frac{\partial^{2}}{\partial z^{2}}-\frac{1}{V_{\mathrm{A}}^{2}} \frac{\partial^{2}}{\partial t^{2}} \\
\mathcal{L}_{\mathrm{i}}=\frac{\partial^{2}}{\partial z^{2}}-\frac{1}{V_{\mathrm{i}}^{2}} \frac{\partial^{2}}{\partial t^{2}}, & \mathcal{L}_{\mathrm{e}}=\frac{\partial^{2}}{\partial z^{2}}-\frac{1}{V_{\mathrm{e}}^{2}} \frac{\partial^{2}}{\partial t^{2}}
\end{array}
$$

$C_{\mathrm{k}}$ is the fast kink speed determined by

$$
\frac{2}{C_{\mathrm{k}}^{2}}=\frac{1}{V_{\mathrm{i}}^{2}}+\frac{1}{V_{\mathrm{e}}^{2}}, \Rightarrow C_{\mathrm{k}}^{2}=\frac{2 B_{0}^{2}}{\mu_{0}\left(\rho_{\mathrm{i}}+\rho_{\mathrm{e}}\right)},
$$

where $B_{0}$ is the (constant) magnitude of the equilibrium magnetic field, and $\mu_{0}$ the magnetic permeability of free space. In our analysis, we use the equation for kink oscillations in a straight magnetic tube, derived in Paper I, using the thin tube approximation. The thin tube approximation means that the $z$ component of the induction equation reduces to (3) to leading order in a series expansion in powers of the radial coordinate. The plasma is only incompressible to leading order. Paper I considers a cold plasma in the presence of background flow but here we consider the static background case.

Equations (3)-(5) are solved inside the tube $(r<R-l / 2$ with $\xi_{r}$ and $\xi_{\varphi}$ only functions of $z$ and $t$ ) and in the external region 
A. W. Hood et al.: Damping of kink waves by mode coupling. I.

( $r>R+l / 2$ with $\xi_{r}$ and $\xi_{\varphi}$ proportional to $1 / r^{2}$ in the thin tube limit). The external solutions are expressed in terms of the internal solutions by integrating the solutions to the equations across the transition layer $(R-l / 2<r<R+l / 2)$. We define the internal solutions, using the thin tube approximation, as

$$
\begin{aligned}
\xi_{r}(r, z, t) & =\eta(z, t), \quad \xi_{\varphi}(r, z, t)=\eta(z, t), \\
\frac{\mu_{0} P_{\mathrm{i}}}{B_{0}^{2}} & =r\left(\frac{\partial^{2} \eta}{\partial z^{2}}-\frac{1}{V_{\mathrm{i}}^{2}} \frac{\partial^{2} \eta}{\partial t^{2}}\right) \equiv r \mathcal{L}_{\mathrm{i}} \eta .
\end{aligned}
$$

Following Paper I and the brief derivation in the Appendix (see Sect. A.2 and Eq. (A.6)), we obtain the equation governing the propagation of the radial component of the fast kink mode, $\eta(z, t)$, namely

$\mathcal{L}_{\mathrm{k}} \eta \equiv \frac{\partial^{2} \eta}{\partial z^{2}}-\frac{1}{C_{\mathrm{k}}^{2}} \frac{\partial^{2} \eta}{\partial t^{2}}=-\mathcal{M}$.

The right-hand side of Eq. (10) describes the damping of the fast kink mode. When the right-hand side is zero, the kink wave propagates undamped from the photospheric boundary. When $\mathcal{M}$ is non-zero inside the transition layer, the energy in the kink wave (as identified by $\eta$ ) is converted into the resonant Alfvén mode (as identified by the spatial growth in amplitude of $\xi_{\varphi}$ at the location where the value of the variable Alfvén speed equals the kink speed $C_{\mathrm{k}}$, i.e. where $\left.V_{\mathrm{A}}\left(r_{s}\right)=C_{\mathrm{k}}\right)$.

The quantity $\mathcal{M}$ on the right-hand side of Eq. (10) is given by (see Appendix, Eq. (A.6))

$\mathcal{M}=-\frac{\ell}{4 R} \mathcal{L}_{\mathrm{i}} \eta+\frac{\ell}{4 R} \mathcal{L}_{\mathrm{e}} \eta+\frac{\mu_{0} \delta P}{2 R B_{0}^{2}}+\frac{1}{2} \mathcal{L}_{\mathrm{e}} \delta \xi_{r}$,

where $\delta P=P_{\mathrm{e}}-P_{\mathrm{i}}$ and $\delta \xi_{r}=\xi_{\mathrm{e}}-\eta$ are the jumps of $P$ and $\xi_{r}$ across the transition layer.

In what follows, we use the thin tube thin boundary (TTTB) approximation, and so have $\epsilon=l / R \ll 1$. Hence, the leading order approximation to $\mathcal{M}$, with respect to $\epsilon$, is given in the Appendix (see Eq. (A.11)) and the propagating kink mode equation can be expressed as

$$
\mathcal{L}_{\mathrm{k}} \eta=-\frac{1}{2 R} \mathcal{L}_{\mathrm{e}} \int_{R-l / 2}^{R+l / 2} \xi_{\varphi} \mathrm{d} r
$$

\section{Derivation of the governing equation}

To progress, the integral involving $\xi_{\varphi}$ in Eq. (12) must be expressed in terms of $\eta$. Hence, we need to obtain the solution for $\xi_{\varphi}$ in the transition layer. Thus, we must solve Eq. (5), where the right hand side is replaced by the leading order solution, i.e. with $P$ replaced by $P_{\mathrm{i}}$ and $r$ by $R$. Next we use Eq. (A.2), from the Appendix, to give to leading order

$\mathcal{L} \xi_{\varphi}=\mathcal{L}_{\mathrm{i}} \eta$

Below, we assume that the photospheric driver at $z=0$ excites a linearly polarized kink wave with frequency $\omega$ propagating in the positive $z$-direction. In the absence of the inhomogeneous annulus, the solution to Eq. (12) describes a constant amplitude, propagating wave for the internal displacement that propagates with the speed $C_{\mathrm{k}}$. The perturbations of all variables, in this case, are functions of $T=t-z / C_{\mathrm{k}}$ only and, for a fixed frequency $\omega$, have the form $\mathrm{e}^{\mathrm{i} \omega T}$. Due to the transition layer, the amplitude of the internal displacement, $\eta$, is resonantly damped and for $\epsilon \ll 1$ the lengthscale for the damping will be much longer than the wavelength $L=2 \pi C_{\mathrm{k}} / \omega$ of the undamped kink wave. We do
Table 1. Definitions of variables and parameters used in this paper.

\begin{tabular}{lc}
\hline \hline Parameter/variable & Definition \\
\hline$\epsilon$ & $l / R$ \\
$\chi$ & $C_{\mathrm{k}} / V_{\mathrm{A}}$ \\
$\kappa$ & $\left(\rho_{\mathrm{i}}-\rho_{\mathrm{e}}\right) /\left(\rho_{\mathrm{i}}+\rho_{\mathrm{e}}\right)$ \\
$X$ & $(r-R) /(l / 2)$ \\
$s$ & $k z$ \\
$Z$ & $\kappa k z / 2$ \\
$\omega T$ & $\omega t-k z$ \\
\hline
\end{tabular}

not prescribe the relationship between the wavelength and the characteristic scale of the amplitude variation, but only assume that this scale is much larger than $L$. Inside the transition layer we define a stretched radial coordinate, namely

$X=\frac{r-R}{l / 2}$

The definitions of important variables used in this paper are given in Table 1.

Thus, we look for solutions for $\eta$ of the form

$\eta(z, t)=\tilde{\eta}(z) \mathrm{e}^{\mathrm{i} \omega T}=\tilde{\eta}(z) \mathrm{e}^{\mathrm{i} \omega\left(t-z / C_{\mathrm{k}}\right)}=\tilde{\eta}(z) \mathrm{e}^{\mathrm{i}(\omega t-k z)}$,

where $k=\omega / C_{\mathrm{k}}$, and assume

$\frac{L}{\tilde{\eta}} \frac{\mathrm{d} \tilde{\eta}}{\mathrm{d} z} \ll 1$.

To keep the expressions as simple as possible, we restrict our attention to the linear density profile. Thus, inside the transition layer,

$\rho=\frac{1}{2}\left[\rho_{\mathrm{i}}+\rho_{\mathrm{e}}-\left(\rho_{\mathrm{i}}-\rho_{\mathrm{e}}\right) X\right]$.

In addition, we must specify boundary conditions for $\eta$ and $\xi_{\varphi}$ on the driven boundary at $z=0$. We assume $\tilde{\eta}(0)=a$ and

$\xi_{\varphi}(r, 0, t)=a \operatorname{sgn}(-X) \mathrm{e}^{\mathrm{i} \omega t}=\left\{\begin{array}{cc}a \mathrm{e}^{\mathrm{i} \omega t}, & -1<X<0, \\ -a \mathrm{e}^{\mathrm{i} \omega t}, & 0<X<1 .\end{array}\right.$

From the integral of Eq. (3), so that $r \xi_{r}=\int \xi_{\varphi} \mathrm{d} r$ and continuity of $\xi_{r}$, the leading order boundary condition for $\xi_{r}$ on $z=0$ is $\xi_{r}(r, z=0, t)=a \mathrm{e}^{\mathrm{i} \omega t}$. This choice matches the form of the undamped kink mode. We remind the reader that terms proportional to $\epsilon$ are dropped to leading order.

The solution to (13) is obtained by the method of Variations of Parameters (Boyce \& DiPrima 2008). Thus, we set

$\xi_{\varphi}=\alpha(r, z) \mathrm{e}^{\mathrm{i} \omega\left(t-z / V_{\mathrm{A}}\right)}+\beta(r, z) \mathrm{e}^{\mathrm{i} \omega\left(t+z / V_{\mathrm{A}}\right)}$,

where $\alpha(r, z)$ and $\beta(r, z)$ are to be determined. We define the ratio of the kink speed to the Alfvén speed as $\chi=C_{\mathrm{k}} / V_{\mathrm{A}}$.

Hence, $\chi=\sqrt{1-\kappa X}$ where $\kappa=\left(\rho_{\mathrm{i}}-\rho_{\mathrm{e}}\right) /\left(\rho_{\mathrm{i}}+\rho_{\mathrm{e}}\right)$. Substituting these results into Eq. (13), we have the pair of equations

$$
\begin{gathered}
\frac{\partial \alpha}{\partial z} \mathrm{e}^{\mathrm{i}(\omega t-k \chi z)}+\frac{\partial \beta}{\partial z} \mathrm{e}^{\mathrm{i}(\omega t+k \chi z)}=0, \\
-\chi \frac{\partial \alpha}{\partial z} \mathrm{e}^{\mathrm{i}(\omega t-k \chi z)}+\chi \frac{\partial \beta}{\partial z} \mathrm{e}^{\mathrm{i}(\omega t+k \chi z)}=-\mathrm{i} k \kappa \tilde{\eta}(z) \mathrm{e}^{\mathrm{i} \omega T},
\end{gathered}
$$


and the solutions to these equations are

$\alpha=C_{1}(X)+\frac{\mathrm{i} k \kappa}{2 \chi} \int_{0}^{z} \tilde{\eta}(u) \mathrm{e}^{-\mathrm{i} k(1-\chi) u} \mathrm{~d} u$,

$\beta=C_{2}(X)-\frac{\mathrm{i} k \kappa}{2 \chi} \int_{0}^{z} \tilde{\eta}(u) \mathrm{e}^{-\mathrm{i} k(1+\chi) u} \mathrm{~d} u$.

When deriving the second equation in Eq. (19), we neglect the small terms containing the derivatives of $\tilde{\eta}(z)$ in the right-hand side. Since the wavelength associated with $\mathrm{e}^{-\mathrm{i} k(1+\chi) z}$ is much less that the damping length of $\eta$, we can integrate by parts (or equivalently average over one wavelength) the expression for $\beta$ and get, approximately,

$\beta=C_{2}(X)-\frac{a \kappa}{2 \chi(1+\chi)}+\frac{\kappa}{2 \chi(1+\chi)} \tilde{\eta}(z) \mathrm{e}^{-\mathrm{i} k(1+\chi) z}$.

To eliminate any downward propagating waves, we choose

$C_{2}(X)=\frac{a \kappa}{2 \chi(1+\chi)}$

so that

$\beta \mathrm{e}^{\mathrm{i}(\omega t+k \chi z)}=\frac{\kappa}{2 \chi(1+\chi)} \tilde{\eta}(z) \mathrm{e}^{\mathrm{i} \omega T}$.

Hence, there is no contribution from downward propagating waves, as expected, and only the term due to the upward propagating kink wave remains.

Integration by parts cannot be used for simplifying $\alpha$ near $X=0$, since the wavelength of the integrand, $2 \pi / k(1-\chi)$, is no longer less than the damping length. However, we can use that approach at $X=-1$ (or equivalently at $r=R-l / 2$ ) to confirm that $\xi_{\varphi}=\tilde{\eta}(z) \mathrm{e}^{\mathrm{i} \omega T}$ there.

Finally, we need to choose $C_{1}(X)$ so that the boundary condition at $z=0$, Eq. (17), is satisfied. Hence,

$C_{1}(X)=a \operatorname{sgn}(-X)-\frac{a \kappa}{2 \chi(1+\chi)}$.

This is the only place where the spatial form of the photospheric driver appears, namely the first term on the right hand side of Eq. (25). Thus, the solution for $\xi_{\varphi}$ is

$$
\begin{aligned}
\xi_{\varphi}= & \left\{\left(a \operatorname{sgn}(-X)-\frac{a \kappa}{2 \chi(1+\chi)}\right.\right. \\
& \left.+\frac{\mathrm{i} k \kappa}{2 \chi} \int_{0}^{z} \tilde{\eta}(u) \mathrm{e}^{-\mathrm{i} k(1-\chi) u} \mathrm{~d} u\right) \mathrm{e}^{\mathrm{i} k(1-\chi) z} \\
& \left.+\frac{\kappa}{2 \chi(1+\chi)} \tilde{\eta}(z)\right\} \mathrm{e}^{\mathrm{i} \omega T} .
\end{aligned}
$$

There are four terms in the expression for $\xi_{\varphi}$, with the first term dependent on the radial form of the boundary condition at $z=0$. The exponential factor for the undamped kink mode, $\mathrm{e}^{\mathrm{i} \omega T}$, has been taken outside the curly brackets, leaving a complex function of $k z, X$ and $\tilde{\eta}$ inside.

Next, we must integrate Eq. (26) across the transition layer. This is done term by term in the Appendix. The final equation governing the propagation and damping of the kink mode, on using Eq. (15) for the form of $\eta$, is

$$
\begin{aligned}
-2 \mathrm{i} \frac{\mathrm{d} \tilde{\eta}}{\mathrm{d} s}+ & \frac{\mathrm{d}^{2} \tilde{\eta}}{\mathrm{d} s^{2}}=\frac{\epsilon K}{4} \int_{-1}^{1} \xi_{\varphi} \mathrm{d} X=\frac{\epsilon K}{4}\{F(s) \\
& \left.+\int_{0}^{s} \tilde{\eta}(u) g(s-u) \mathrm{d} u+\tilde{\eta}(s) \ln \left[\frac{p_{4}}{p_{3}}\right]\right\}
\end{aligned}
$$

on cancelling the common factor of $\mathrm{e}^{\mathrm{i} \omega T}$ and setting $s=k z$. In deriving Eq. (27), we have only applied the operator $\mathcal{L}_{\mathrm{e}}$ to the multiplier $\mathrm{e}^{\mathrm{i} \omega T}$. The additional terms are small for $s$ greater than unity. They are also small for $\kappa \ll 1$. The inhomogeneous term is given by

$$
\begin{aligned}
F(s)= & -\frac{4 a}{\kappa s^{2}}-\mathrm{i} \frac{4 a}{\kappa s}+\frac{2 a}{\kappa s^{2}}\left(\mathrm{e}^{\mathrm{i} s p_{1}}+\mathrm{e}^{\mathrm{i} s p_{2}}\right) \\
& +\mathrm{i} \frac{2 a}{\kappa s}\left(\sqrt{1-\kappa} \mathrm{e}^{\mathrm{i} s p_{1}}+\sqrt{1+\kappa} \mathrm{e}^{\mathrm{i} s p_{2}}\right) \\
& +a \mathrm{e}^{\mathrm{i} 2 s}\left[\mathrm{Ci}\left(s p_{3}\right)-\mathrm{Ci}\left(s p_{4}\right)-\mathrm{iSi}\left(s p_{3}\right)+\mathrm{iSi}\left(s p_{4}\right)\right],
\end{aligned}
$$

and

$g(s-u)=\frac{\mathrm{e}^{\mathrm{i}(s-u) p_{1}}-\mathrm{e}^{\mathrm{i}(s-u) p_{2}}}{s-u}$.

We define, for later use, the complex function

$G(s)=\int_{0}^{s} g(s-u) \mathrm{d} u$.

In Eqs. (27)-(29), we have used the shorthand notation,

$p_{1}=1-\sqrt{1-\kappa}, p_{2}=1-\sqrt{1+\kappa}$,

$p_{3}=1+\sqrt{1-\kappa}, p_{4}=1+\sqrt{1+\kappa}$,

and $\mathrm{Ci}(x)$ and $\operatorname{Si}(x)$ are the cosine integral and the sine integral, as defined in Abramowitz \& Stegun (1965) and the Appendix.

When $\kappa$ is not too large, i.e. less than about $1 / 2$, we can use the approximations $p_{1} \approx \kappa / 2$ and $p_{2} \approx-\kappa / 2$ and express $F(s)$ as

$F(s)=-2 \mathrm{i} a\left(\frac{1-\cos Z}{Z}\right)+O(\kappa)$,

where $Z=\kappa k z / 2$. This approximation is valid for $\kappa \ll Z \ll 1 / \kappa$. A more accurate representation of $F$, by taking an extra term in the $\kappa$ expansions of $p_{1}$ and $p_{2}$ is given by

$F(s)=-2 \mathrm{i} a\left(\frac{1-\cos Z \cos \left(\frac{\kappa Z}{4}\right)}{Z}\right)+O(\kappa)$,

by considering variations over the slower spatial scale given by $\kappa Z / 4=\kappa^{2} k z / 8$. Note that the Taylor series expansion for small $\kappa Z / 4$ gives the same approximation as Eq. (32). The difference between the two approximations only occurs for large $\kappa Z / 4$ and remains bounded. In addition, the expansion for small $Z$ agrees with small $k z$ expansion discussed below. Hence, the approximation given by Eq. (32) remains accurate for $0<Z<4 / \kappa$.

\section{Investigation of damping}

In this section we investigate the spatial form of the damping of the kink mode. Firstly, we show that for small distances the amplitude decays Gaussianally and that this behaviour is not due to the specific form of the photospheric driver. Next we investigate a simple expansion in powers of $\epsilon$, the ratio of the width of the transition layer to the radius of the flux tube. This illustrates that the simple expansion breaks down once a certain distance is reached. Finally, we use an expansion valid for small density ratios to derive a relatively simple looking equation to describe the damping. This form of equation allows us to investigate commonly used assumptions in the next subsection and demonstrates the importance of the various terms involved. 
A. W. Hood et al.: Damping of kink waves by mode coupling. I.

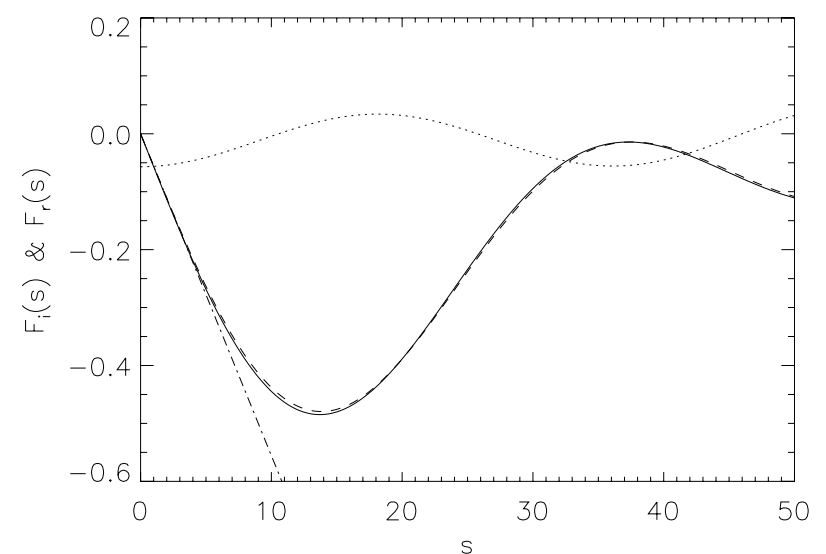

Fig. 1. Imaginary part of $F(s)$ shown as a solid curve and small $z$ expansion shown as a dot-dashed curve, for $\kappa=1 / 3$. The real part of $F(s)$ is the dotted curve. The approximation to the imaginary part of $F(s)$ given by (33) is the dashed curve.

\subsection{Small $\mathrm{kz}$}

Let us use Eq. (27) to study the properties of propagating kink waves. We start our analysis by investigating the solution of this equation for small $k z$. In the Appendix, we show that the expansion of $F(s)$ on the right-hand side of Eq. (27) is given by, (see Sects. A.4.1 and A.4.2),

$$
\begin{aligned}
F(s) \approx & \mathrm{i} s \mathcal{B}-a(1+2 \mathrm{i} s) \ln \left[\frac{1+\sqrt{1+\kappa}}{1+\sqrt{1-\kappa}}\right] \\
& +\mathrm{i} s a(\sqrt{1+\kappa}-\sqrt{1-\kappa}),
\end{aligned}
$$

where

$\mathcal{B}=\frac{2}{3 \kappa} a\left(2-(1+\kappa)^{3 / 2}-(1-\kappa)^{3 / 2}\right)$.

For example, for $\kappa=1 / 3$, the linear approximation provides a good fit to both $F(s)$ and the coefficient of $\tilde{\eta}$ out to $s \approx 5$ (see Fig. 1). In this case, small $s$ means $0 \leq s<5$. Therefore, we can express Eq. (27) as

$$
\begin{aligned}
-2 \mathrm{i} \frac{\mathrm{d} \tilde{\eta}}{\mathrm{d} s}+\frac{\mathrm{d}^{2} \tilde{\eta}}{\mathrm{d} s^{2}}= & \frac{\epsilon \kappa}{4}\left\{\mathrm{i} s \mathcal{B}-2 \mathrm{i} s a \ln \left[\frac{1+\sqrt{1+\kappa}}{1+\sqrt{1-\kappa}}\right]\right. \\
& +2 \mathrm{i} s a[\sqrt{1+\kappa}-\sqrt{1-\kappa}] \\
& +\mathrm{i} \int_{0}^{s}(\tilde{\eta}(u)-a)[\sqrt{1+\kappa}-\sqrt{1-\kappa}] \mathrm{d} u \\
& \left.+(\tilde{\eta}(s)-a) \ln \left[\frac{1+\sqrt{1+\kappa}}{1+\sqrt{1-\kappa}}\right]\right\} .
\end{aligned}
$$

When $\kappa$ is not too close to unity, $\kappa<1$, Eq. (36) can be approximated by

$$
\begin{aligned}
-2 \mathrm{i} \frac{\mathrm{d} \tilde{\eta}}{\mathrm{d} s}+ & \frac{\mathrm{d}^{2} \tilde{\eta}}{\mathrm{d} s^{2}}= \\
& \frac{\epsilon \kappa^{2}}{8}\left\{(\tilde{\eta}(s)-a)+2 \mathrm{i} \int_{0}^{s}(\tilde{\eta}(u)-a) \mathrm{d} u+a \mathrm{i} s\right\} .
\end{aligned}
$$

This form of the equation is used to illustrate the method of analysis with the result for more general $\kappa$ listed below. Since $\tilde{\eta}=a$ when $\epsilon=0$, we set $\tilde{\eta}-a=\epsilon \tilde{\eta}_{1}(s)$ and, in the weak damping limit and for small values of $s$, terms containing $\tilde{\eta}-a$ on the right hand side of Eq. (36) can be neglected. Hence, the $O(\epsilon)$ equation is

$-2 \mathrm{i} \frac{\mathrm{d} \tilde{\eta}_{1}}{\mathrm{~d} s}+\frac{\mathrm{d}^{2} \tilde{\eta}_{1}}{\mathrm{~d} s^{2}}-\frac{\kappa^{2}}{8}\{a \mathrm{i} s\}=0$.

The solution is to this equation is the complementary function and a particular integral. Since it has to satisfy the boundary condition $\tilde{\eta}(0)=a$, it contains only one arbitrary constant. To determine this constant, we once again use the condition that there is no downward propagating wave. As a result we obtain

$\tilde{\eta}=a\left(1-\frac{\epsilon \kappa^{2} s^{2}}{32}+\mathrm{i} \frac{\epsilon \kappa^{2} s}{32}\right)+O\left(\epsilon^{2}\right)$.

It is common in considering the effect of weak damping, where $\mathrm{d} \tilde{\eta} / \mathrm{d} s=O(\epsilon)$, to assume that the second derivative will be $O\left(\epsilon^{2}\right)$, i.e. smaller than the first derivative. However, this is not the case, as shown by Eq. (39). Instead the imaginary term in Eq. (39) results from keeping the second derivative. The amplitude of $\tilde{\eta}$ is, however,

$1-\frac{\epsilon K^{2} s^{2}}{32}+O\left(\epsilon^{2}\right)$

and this can be obtained directly from Eq. (37) by dropping the second derivative. Thus, the second derivative term modifies the phase of the weakly damped kink mode.

Repeating the above method for general $\kappa$, we can show that, for small $s$,

$\tilde{\eta} \approx a\left[1-\epsilon q(\kappa)(s)^{2}\right]$

where

$$
\begin{aligned}
q(\kappa)= & \frac{1}{24}[2-(1-2 \kappa) \sqrt{1+\kappa}-(1+2 \kappa) \sqrt{1-\kappa}] \\
& +\frac{\kappa}{8} \ln \frac{1+\sqrt{1-\kappa}}{1+\sqrt{1+\kappa}} .
\end{aligned}
$$

It is worth noting that

$q(\kappa) \approx \frac{\kappa^{2}}{32}$

with the accuracy better than $2.5 \%$ for $0<\kappa<1$. This approximation for $q$ can be used even for values of $\kappa$ close to unity. For example, when the density contrast is 10 so that $\kappa=9 / 11$, the maximum error in replacing Eq. (41) by the simpler expression of Eq. (42) is less than $1.6 \%$.

To clearly highlight the behaviour of the damping, we investigate the logarithm of $\tilde{\eta}$ so that, for example, $\mathrm{e}^{-z / L}$ would appear as a straight line. For our expansion given by Eq. (40), we have $\ln (\tilde{\eta} / a) \approx-\epsilon q(\kappa) s^{2}$, where $s=k z$. This is consistent with

$\ln (\eta / a) \approx\left(-\frac{\epsilon K^{2} s^{2}}{32}\right)$

but there are other functions which have the same initial terms in their expansion. For example,

$\tilde{\eta} \approx \frac{a}{2}\left\{1+\exp \left(-\frac{\epsilon \kappa^{2} s^{2}}{16}\right)\right\}=\frac{a}{2}\left\{1+\exp \left(-\frac{z^{2}}{L_{\mathrm{g}}^{2}}\right)\right\}$,

where

$L_{\mathrm{g}}^{2}=\frac{16}{\epsilon \kappa^{2} k^{2}}$, 


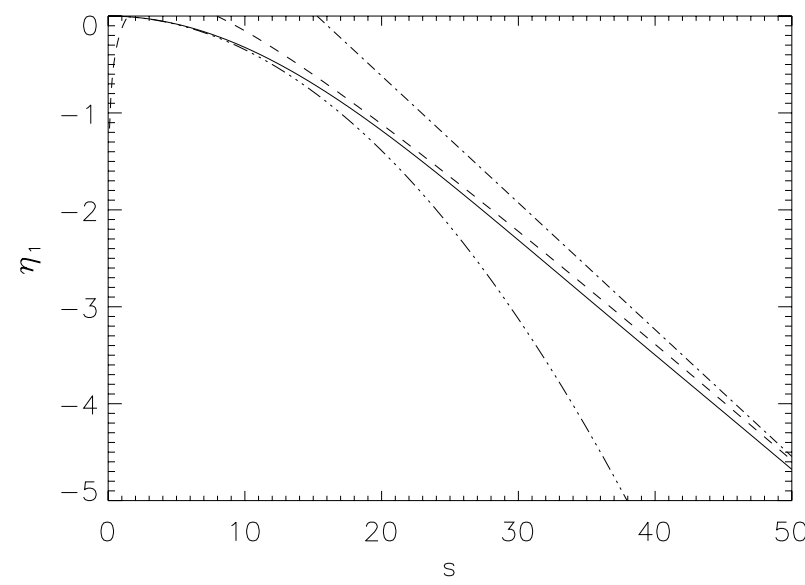

Fig. 2. $\tilde{\eta}_{1}$ is shown as a function of $s$ as a solid curve, for $\kappa=1 / 3$. The triple dot-dashed curve is the approximation $-(\kappa s)^{2} / 32$, the dot-dashed curve is $-\pi(\kappa s) / 8+2$ and the dashed curve is $-\pi(\kappa s) / 8+\ln (\kappa s / 2) / 2+0.9$. Note that the function is initially Gaussian for small $s$ but, although modified by the logarithm term, it is approximately linear for large $s$.

is a slightly better fit to the numerical solution provided $s$ is not too large. Here not too large means up to a distance of approximately a wavelength divided by $\kappa$ from the boundary driver. Since the long wavelength limit is used, this can be a significant distance from the location of the driver.

Thus, the amplitude of $\tilde{\eta}$ decreases Gaussianally for small $s$. Although the series given by Eq. (40) is the correct expression for small $s$, this can be expressed by a number of different functional forms that have the same expansion for small $s$.

\subsection{Expansion in powers of $\epsilon$}

Before progressing, we study the approximations to $F(s)=$ $F_{\mathrm{r}}(s)+\mathrm{i} F_{\mathrm{i}}(s)$. Figure 1 shows that the imaginary part of $F(s)$, $F_{\mathrm{i}}(s)$, dominates $F_{\mathrm{r}}(s)$, for $0<s<30$, and the small $s$ expansion is valid out to $s=5$. Note that Eq. (33) provides a good fit to $F_{\mathrm{i}}(s)$ for $0<s<50$, as seen in Fig. 1 . Now we look to express $\tilde{\eta}$ in powers of $\epsilon$ as $\tilde{\eta}=a+\epsilon \tilde{\eta}_{1}(s)+O\left(\epsilon^{2}\right)$. Hence, $\ln \tilde{\eta}=\ln a+\epsilon \tilde{\eta}_{1}(s)+O\left(\epsilon^{2}\right)$. Since both the imaginary parts of $F(s)$ and $G(s)$ are larger than the respective real parts, we drop the real parts. Dropping the second derivative, we have

$-2 \frac{\mathrm{d} \tilde{\eta}_{1}}{\mathrm{~d} s}=\frac{\kappa}{4}\left(F_{\mathrm{i}}(s)+a G_{\mathrm{i}}(s)\right)$.

Hence,

$\tilde{\eta}_{1}(s)=-\frac{\kappa}{8} \int_{0}^{s}\left(F_{\mathrm{i}}(u)+a G_{\mathrm{i}}(u)\right) \mathrm{d} u$.

This is shown is Fig. 2. Our simple expansion in powers of $\epsilon$ must break down whenever $\epsilon \tilde{\eta}_{1}$ becomes of order unity. This secular behaviour suggests that there is a slower lengthscale associated with the damping of the kink mode. However, this slow scale is not simply a linear combination of $\epsilon$ and $z$.

Thus, the expansion in powers of $\epsilon$ is only valid for small enough distances, such that $\epsilon \tilde{\eta}_{1} \ll 1$. For large distances, this expansion will always break down. However, the distance where it begins to break down does depend strongly on $\epsilon$. For small enough $\epsilon$, we can clearly see how the form of the damping is initially Gaussian in character but it switches to an almost linear form further up. If $\epsilon$ is larger, but still less than unity, the expansion will be valid for the Gaussian part only.

\subsection{Expansion in powers of $\kappa$}

Returning to the kink mode Eq. (27), we expand the right hand side in powers of $\kappa$. Defining the new independent variable $Z=$ $\kappa k z / 2$, the equation is

$$
\begin{aligned}
& -\mathrm{i} \frac{\mathrm{d} \tilde{\eta}}{\mathrm{d} Z}+\frac{\kappa}{4} \frac{\mathrm{d}^{2} \tilde{\eta}}{\mathrm{d} Z^{2}}= \\
& -\mathrm{i} \frac{\epsilon}{2}\left\{\frac{a(1-\cos Z)}{Z}-\int_{0}^{Z} \tilde{\eta}(u) \frac{\sin (Z-u)}{Z-u} \mathrm{~d} u\right\}+O(\kappa) .
\end{aligned}
$$

Hence, the second order derivative can be neglected to leading order in $\kappa$ and the weak density variation assumption $\kappa \ll 1$ leads to

$\frac{\mathrm{d} \tilde{\eta}}{\mathrm{d} Z}=\frac{\epsilon}{2}\left\{\frac{a(1-\cos Z)}{Z}-\int_{0}^{Z} \tilde{\eta}(u) \frac{\sin (Z-u)}{Z-u} \mathrm{~d} u\right\}$.

Equation (47) is the important equation that can be used to investigate the spatial damping of the propagating kink mode, whenever the density contrast is not too large. For example, good agreement is found with the numerical solutions (see Sect. 5) whenever $\rho_{\mathrm{i}} / \rho_{\mathrm{e}} \leq 3$ or equivalently $\kappa \leq 1 / 2$. The advantage of Eq. (47) over the full expression in Eq. (27) is its relative simplicity. This equation can be solved numerically. We can use it also to investigate how some standard approximations compare with the full solution. In addition, the inhomogeneous term in Eq. (47) is derived directly from the imposed form of the photospheric driver. Changing the driver changes this one term. However, as we will see below, neglecting this term does not change the conclusion that the damping is Gaussian in nature over the first few wavelengths. It is just that the rate of the Gaussian damping is different.

\subsubsection{Expansion in powers of $\epsilon$}

Next, we can expand $\tilde{\eta}$ in powers of $\epsilon$ and obtain

$\tilde{\eta}=a+\frac{\epsilon a}{2}\left\{\int_{0}^{Z} \frac{1-\cos u}{u} \mathrm{~d} u-\int_{0}^{Z} \int_{0}^{u} \frac{\sin s}{s} \mathrm{~d} s \mathrm{~d} u\right\}$.

Evaluating the integrals, we have

$\tilde{\eta}=a+\frac{\epsilon a}{2}\{\gamma+1+\ln Z-\operatorname{Ci}(Z)-Z \operatorname{Si}(Z)-\cos Z$,

where $\gamma \approx 0.5772$ is Euler's constant and $\mathrm{Ci}(Z)$ and $\mathrm{Si}(Z)$ are the Cosine and Sine integrals respectively (Abramowitz \& Stegun 1965). We can approximate $\tilde{\eta}$ by

$a-\frac{\epsilon a}{2}\left(\frac{Z^{2}}{4}-\frac{Z^{4}}{288}+\frac{Z^{6}}{21600}\right) \quad$ for $Z<4$,
$a-\frac{\epsilon a}{2}\left(\frac{\pi Z}{2}-\ln Z-1-\gamma+\frac{\cos Z}{Z^{2}}\right)$ for $Z>4$,

where the appropriate asymptotic expansions for $\mathrm{Ci}(Z)$ and $\mathrm{Si}(Z)$ have been used. Note that the $\operatorname{logarithm}$ of $\tilde{\eta}$, when $\epsilon$ is small, is simply

$\ln (\tilde{\eta} / a)=\frac{\epsilon}{2}(\gamma+1+\ln Z-\operatorname{Ci}(Z)-Z \operatorname{Si}(Z)-\cos Z)$.

This form is used when comparing with the numerical solution for small $\epsilon$ in Sect. 5 below. From Eq. (49), we expect $\ln \tilde{\eta}$ to behave like $-\epsilon \pi Z / 4+(\epsilon \ln Z) / 2$ for large $Z$, so that the Terradas et al. (2010) results will slightly over-estimate the damping rate 
due to the neglect of the logarithmic term. The behaviour for small $Z$ is again the same as the real part of Eq. (39).

We remind the reader that, for large $Z$, the expansion in powers of $\epsilon$ will break down whenever the magnitude of $\tilde{\eta}-a$ becomes of order unity. The small $Z$ expansion, however, will remain valid for small $\epsilon$ and $\kappa$.

\subsection{Approximate solutions to Eq. (47)}

Equation (47) can be solved numerically and the results are compared with the full numerical solution to the linear MHD equations (as discussed in Pascoe et al. 2012). This comparison is discussed in Sect. 5. Before that, we can use Eq. (47) to investigate commonly used approximations in the large $z$ limit and compare these approximations with the numerical solution of Eq. (47).

\subsubsection{Approximation 1}

Firstly, consider the behaviour of $\tilde{\eta}(k z)$ for $1 \ll k z \ll \epsilon^{-1}$. The first term on the right-hand side of Eq. (47) is $O(1 / z)$ when compared to the second term. Hence, we neglect the first term. Thus, for large $Z$, we solve

$\frac{\mathrm{d} \tilde{\eta}}{\mathrm{d} Z}+\frac{\epsilon}{2} \int_{0}^{Z} \tilde{\eta}(u) \frac{\sin (Z-u)}{Z-u} \mathrm{~d} u=0 . \quad$ (Approx. 1)

where $Z=\kappa k z / 2$. This approximate equation can be solved by a Laplace transform but it is not straightforward to invert the transform back to physical space. Instead we solve this equation numerically to determine Approximation $1, \tilde{\eta}_{1}(Z)$. Approximation 1 is shown in Fig. 3 as a dashed curve.

\subsubsection{Approximation 2}

Using the mean value theorem, the integral in Eq. (51) can be expressed as

$$
\begin{aligned}
\int_{0}^{Z} \tilde{\eta}(u) \frac{\sin (Z-u)}{Z-u} \mathrm{~d} u= & \tilde{\eta}(z) \int_{0}^{Z} \frac{\sin (Z-u)}{Z-u} \mathrm{~d} u \\
& -\int_{0}^{Z} \tilde{\eta}^{\prime}(c) \sin (Z-u) \mathrm{d} u,
\end{aligned}
$$

where the derivative of $\tilde{\eta}$ is evaluated at $c$ and $u \leq c \leq Z$. Since the derivative of $\tilde{\eta}$ is $O(\epsilon)$, we neglect the second term. Hence, Eq. (51) reduces to

$$
\frac{\mathrm{d} \tilde{\eta}}{\mathrm{d} Z}+\tilde{\eta}(z) \frac{\epsilon}{2} \int_{0}^{Z} \frac{\sin (Z-u)}{Z-u} \mathrm{~d} u=0 . \quad \text { (Approx. 2) }
$$

and this can be solved by an integrating factor to give

$$
\begin{aligned}
\tilde{\eta}_{2}(Z) & =a \exp \left\{-\frac{\epsilon}{2} \int_{0}^{Z} \operatorname{Si}(u) \mathrm{d} u\right\} \\
& =a \exp \left\{-\frac{\epsilon}{2}(Z \operatorname{Si}(Z)+\cos Z-1)\right\} .
\end{aligned}
$$

For large $Z, \operatorname{Si}(Z) \rightarrow \pi / 2$ and so

$\tilde{\eta} \approx a \exp \left\{-\frac{\epsilon \pi \kappa k z}{8}\right\}$

as derived by Terradas et al. (2010). This approximate solution is shown in Fig. 3 as a dot-dashed curve. Despite neglecting the inhomogeneous terms, which arise through the form of the photospheric driver when solving for the resonant Alfvén mode inside

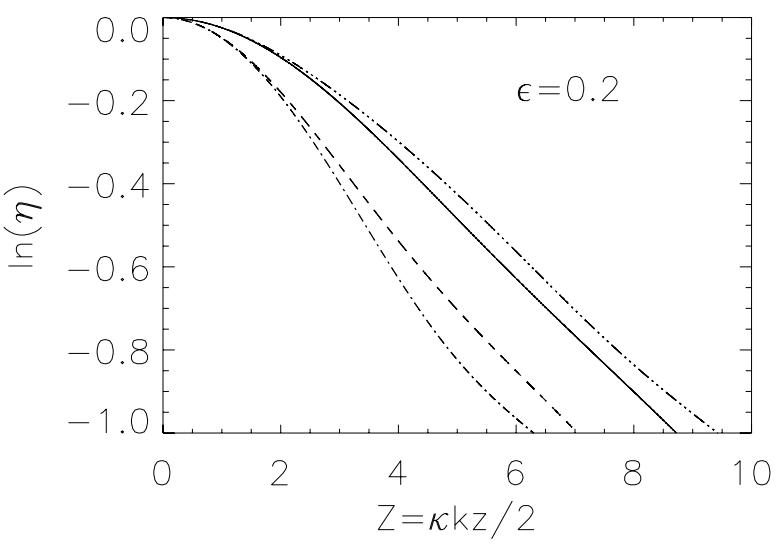

Fig. 3. Different approximations for $\tilde{\eta}$ shown as a function of $Z=$ $\kappa k z / 2$ for $\epsilon=0.2$. The solid curve is the numerical solution to Eq. (47). Approximation 1, Eq. (51), is shown as a dashed curve, Approximation 2 as a dot-dashed curve and Approximation 3 as a triple dot-dashed curve.

the transition layer, this solution also has a Gaussian form for small $Z$ of the form $a \exp \left\{-\epsilon Z^{2} / 4\right\}$ and only takes on the linear exponential damping for large $Z$. So the inhomogeneous term only changes the value of the coefficient of $Z^{2}$ in the Gaussian damping.

\subsubsection{Approximation 3}

Next we re-introduce the inhomogeneous term on the right-hand side of Eq. (47) but take the $\tilde{\eta}(z)$ outside the integral again and investigate

$$
\begin{array}{r}
\frac{\mathrm{d} \tilde{\eta}}{\mathrm{d} Z}+\frac{\epsilon}{2} \tilde{\eta}(z) \int_{0}^{Z} \frac{\sin (Z-u)}{Z-u} \mathrm{~d} u= \\
\frac{\epsilon}{2}\left\{\frac{1-\cos Z}{Z}\right\} .
\end{array}
$$

(Approx. 3)

Again we can use an integrating factor to obtain

$$
\begin{aligned}
\tilde{\eta}_{3}(Z)= & a\left\{\int_{0}^{Z} \frac{\epsilon}{2}\left[\frac{1-\cos u}{u}\right] \exp \left(\frac{\epsilon}{2} \int_{0}^{u} \operatorname{Si}(s) \mathrm{d} s\right) \mathrm{d} u\right. \\
& +1\} \exp \left(-\frac{\epsilon}{2} \int_{0}^{Z} \operatorname{Si}(u) \mathrm{d} u\right) .
\end{aligned}
$$

The first term in curly brackets on the right-hand side is due to the inhomogeneous term, while the second term is the same as Approximation 2. $\tilde{\eta}_{3}(Z)$ is shown as the triple dot-dashed curve in Fig. 3. Note that for small $Z$ this approximation has the form

$$
\begin{aligned}
\tilde{\eta}_{3}(Z) & \approx a\left\{\int_{0}^{Z} \frac{\epsilon}{4} u \mathrm{e}^{\epsilon u^{2} / 4} \mathrm{~d} Z+1\right\} \mathrm{e}^{-\epsilon Z^{2} / 4} \\
& \equiv \frac{a}{2}\left\{1+\mathrm{e}^{-\epsilon Z^{2} / 4}\right\} .
\end{aligned}
$$

As shown above in Eq. (43), this approximate solution has the same Taylor series expansion as the small $z$ expansion derived earlier.

Finally, we can solve Eq. (51) numerically to determine the validity of using the mean value theorem to derive Approximation 3. The numerical result for $\tilde{\eta}$ is shown in Fig. 3 as the solid curve.

There is a significant difference between the approximate solutions $\tilde{\eta}_{1}(Z), \tilde{\eta}_{2}(Z)$ and $\tilde{\eta}_{3}(Z)$ when $Z$ is sufficiently large. The neglect of the inhomogeneous term, Approximations 1 and 2, 
changes the coefficient of the Gaussian term at small $Z$ and results in too much damping. However, it still remains Gaussian in nature. For larger $Z$, Approximation 1 is more or less parallel to the numerical solution to Eq. (47). Approximation 2 damps significantly faster. Approximation 3 includes the inhomogeneous term but the simplifying assumption of taking $\tilde{\eta}$ outside the integral predicts a slower damping rate at large $Z$. However, it does have the correct behaviour for small $Z$.

The best approach in understanding the spatial damping of the kink mode through mode coupling to the Alfvén mode in the transition layer, is to solve Eq. (47) numerically. Keeping in mind the results shown in Fig. 3, the error in using Approximation 3 is not too significant. It has the advantage of having a solution in a closed analytical form. The functions $\mathrm{Si}$ and $\mathrm{Ci}$ are rapidly obtained from computer algebra packages, such as Maple, and it is easy to use a package to numerically integrate the terms in Eq. (54). The small $Z$ expansion, using Eq. (54) gives the correct Gaussian behaviour.

\section{Comparison with numerical results}

The numerical solution to the linear MHD equations remains the most accurate description of the damped kink mode. In this section, the approximate solutions derived (and the methods illustrated) in the previous section are compared with the actual numerical results. Two different numerical codes are used, a second order Lax-Wendroff scheme and a fourth order, finite difference method. The numerical results obtained with the two different methods are consistent and indicate that the results obtained are not dependent on the method used to solve the linear MHD equations. We consider two examples for a small transition layer, namely small $\epsilon(\epsilon<\kappa<1)$ and a small density contrast $(\kappa<\epsilon<1)$.

Figure 4 shows the results for a period of $36 \mathrm{~s}$, a width of the transition layer to radius ratio of 0.2 and a density contrast of 1.3. Thus, we have $\epsilon=0.2$ and $\kappa \approx 0.13$ and the small $\kappa$ Eq. (47) is appropriate. The solution to Eq. (47), namely the amplitude of the kink mode, is shown as a dashed curve, while the solid curve is the full numerical solution for the kink mode, $\xi_{r}$, on the axis, $r=0$. The agreement is extremely good for all distances apart from the leading initial wavelength. To illustrate the actual form of the damping the logarithm of the absolute value of $\xi_{r}$ is also shown. What is clear is how the behaviour of the damping is Gaussian for small distances, as shown in Sect. 4, and switches to almost linear for larger distances. Despite having $\epsilon=0.2$, the TTTB equation provides an extremely good fit to the full solution. This is because the density ratio is only 1.3 and the important parameter $\kappa$ is small. Thus, the damping is weak and Eq. (47) provides a good approximation.

Next we consider the situation where the period is $24 \mathrm{~s}$, which is just long enough for the long wavelength limit to apply, the width of the transition layer to radius ratio is small $(\epsilon=0.05)$ and the density contrast is $2(\kappa=1 / 3)$. Note that the value of $\kappa$ is still quite small and we expect Eqs. (48) and (49) to give a good approximation. The comparison is shown in Fig. 5. As above, the dashed curve in Fig. 5 outlines the amplitude of the kink mode, by solving Eq. (47). In addition, the solid curve is the result of the small $\epsilon$ expansion given by Eq. (48). Both of the approximations match with the numerical solution to the linear MHD equations, showing that, although the small $\kappa$ equation has a relatively simple form, the solution provides excellent agreement with the numerical solution.

Finally, we show the results for a density contrast of 10, pe$\operatorname{riod}$ of $48 \mathrm{~s}$ and $\epsilon=0.05$ in Fig. 6. For these parameters, the thin
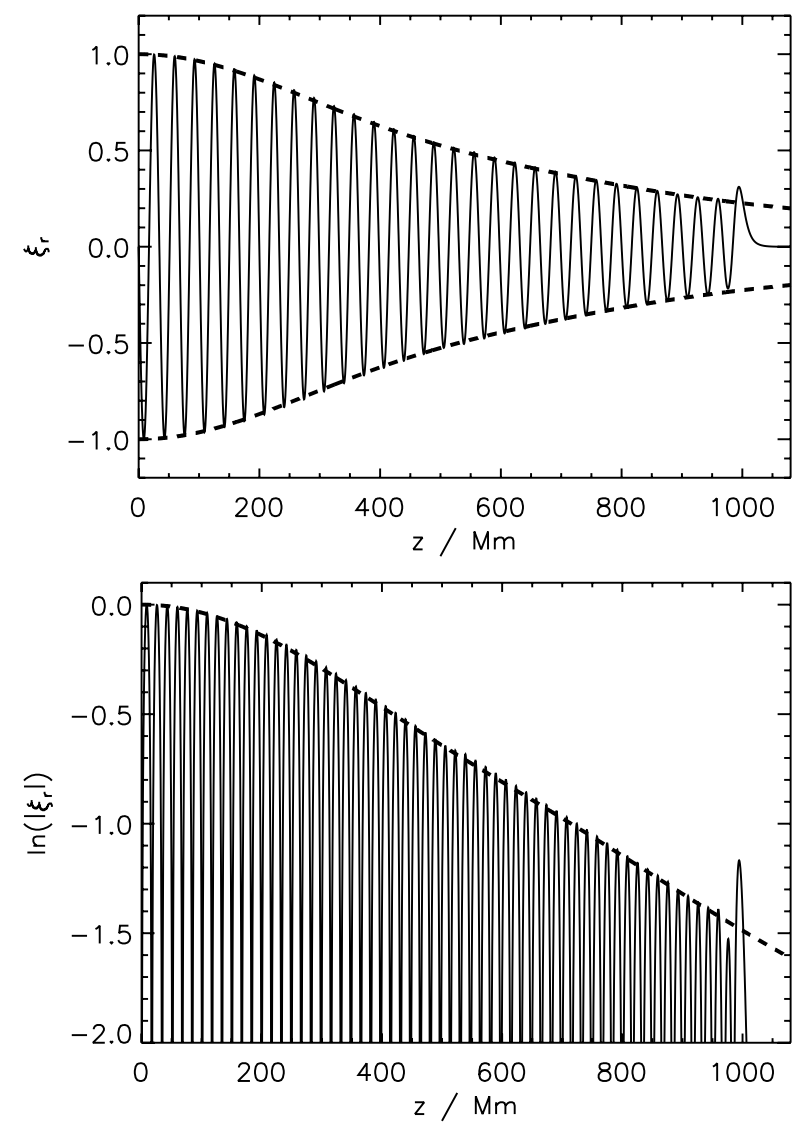

Fig. 4. Amplitude of $\eta=\xi_{r}$ and logarithm of the modulus of $\eta$ at the centre of the tube shown as functions of distance $z$. The solid curve represents the numerical solution, the dashed curve is the numerical solution of (47). The period is $36 \mathrm{~s}$, the ratio of the width of the transition layer to the radius is 0.2 and the density ratio, $\rho_{\mathrm{i}} / \rho_{\mathrm{e}}=1.3$.

tube, thin boundary analysis is still appropriate. However, what is not so clear is whether the small $\kappa$ description is still relevant. From Sect. 4.1, we expect the form of the Gaussian profile to be unaffected by the large density contrast, since the approximation given by Eq. (42), is accurate to better than $2 \%$ for this choice. Hence, the initial Gaussian part still provides an excellent approximation. In addition, at large $z$, the damping will approach the limit predicted by Terradas et al. (2010) and, with the small and large $z$ limits fixed, the approximation given by the solution to Eq. (47) continues to give an excellent fit to the numerical results.

\section{Conclusions}

So which approximations should one use in analysing observations of propagating kink modes? For the magnetic flux tube considered in this paper, if the density contrast is large, then the full damped kink mode equation, Eq. (27), is used. However, if the density contrast is smaller than about 3 , then solutions to the small $\kappa$ equation, Eq. (47), agree with the full numerical results. The solution to Eq. (47) can be approximated by the analytical solution of Approximation 3, Eq. (54), where the Sine Integral, $S i$, is readily computed in various computer algebra packages.

The different approximations used in solving Eq. (47) show clearly that the Gaussian behaviour for small distances is not just due to the form of driving on the boundary. It appears in the homogeneous kink mode equation as well, when the radial profile of $\xi_{r}$ on $z=0$ is completely ignored. However, the form of the 
A. W. Hood et al.: Damping of kink waves by mode coupling. I.
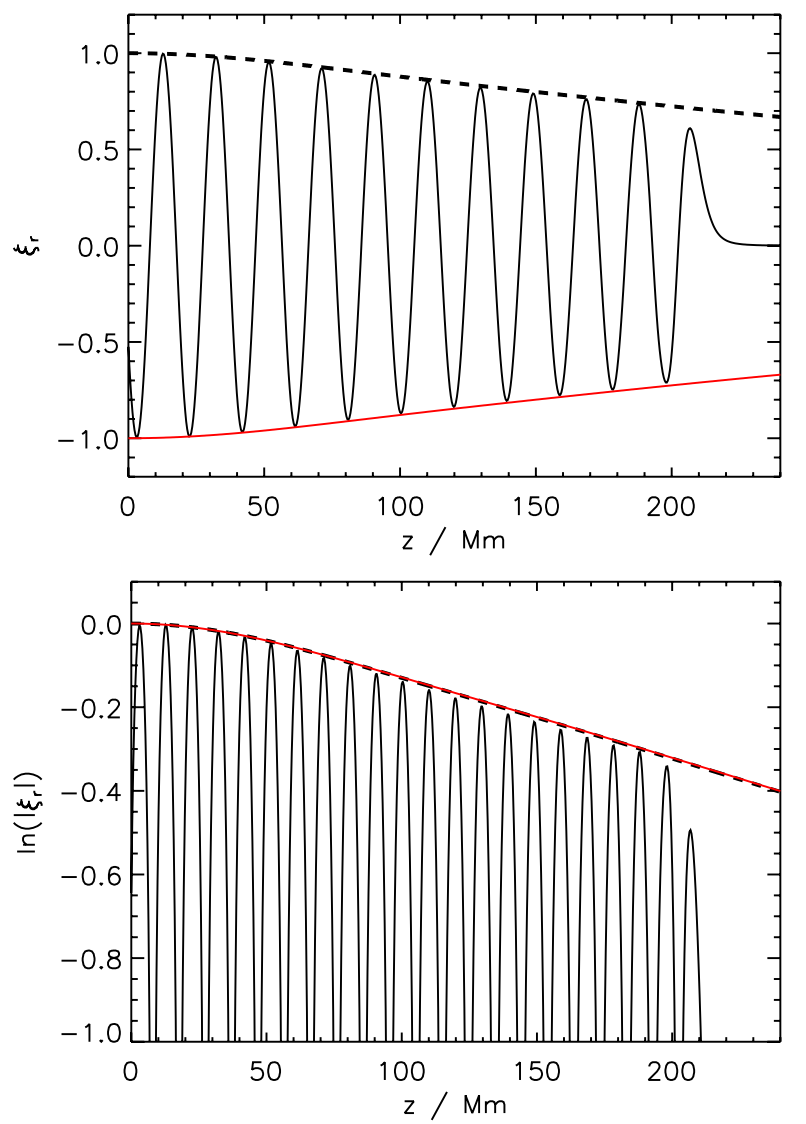

Fig. 5. Amplitude of $\eta=\xi_{r}$ and logarithm of the modulus of $\eta$ at the centre of the tube shown as functions of distance $z$. The solid curve represents the numerical solution using the fourth order scheme, the dashed curve is the numerical solution of Eq. (47) and the red solid curve is the small $\epsilon$ solution given by Eq. (48). The period is $24 \mathrm{~s}$, the width of the transition layer is 0.05 and the density ratio, $\rho_{\mathrm{i}} / \rho_{\mathrm{e}}=2$.

boundary driver does influence the value of the coefficient of the Gaussian term. It is possible to eliminate the Gaussian damping by selecting a very specific photospheric driving profile, that has strong localised variations inside the transition layer. Any smoother profile will result in the Gaussian behaviour noted in this paper.

The nature of the damping of the kink mode changes at larger distances to roughly linear, although there is really a series solution here which varies gently over several wavelengths. The change in character from Gaussian to "almost linear" occurs between the values of $Z=2$ and $Z=4$ and is essentially independent of the width of the transition layer. If $L$ is the wavelength of the undamped kink mode, this change over distance can be expressed in terms of $L$ as $2 L / \pi \kappa$ and $4 L / \pi \kappa$. For a density ratio of 2 , or $\kappa=1 / 3$, the Gaussian behaviour is appropriate for at least 2 to 3 wavelengths, while a density ratio of $1.3(\kappa=0.13)$, on the other hand, it is valid for 5 to 10 wavelengths.

The small- $\kappa$ equation is a relatively simple equation that can be used to investigate the damping of the kink mode and the coupling to the Alfvén mode in the transition layer. While both $\kappa$ and $\epsilon$ should be small, whether $\kappa<\epsilon$ or visa-versa is unimportant. The comparison between the predictions of the small $-\kappa$ equation and the full numerical solution is extremely good for the cases shown. However, a detailed parameter study is necessary to determine how good the small- $\kappa$ assumption is (see Pascoe et al. 2013). This also determines how to use both the Gaussian and
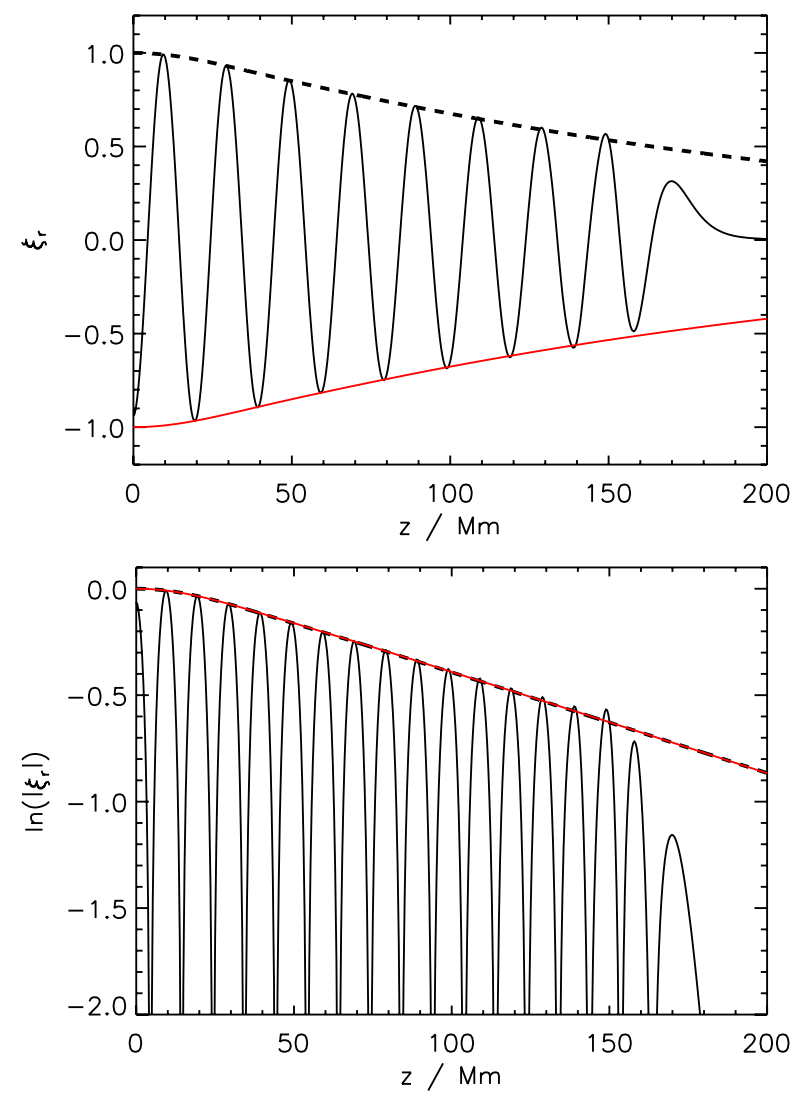

Fig. 6. Line styles as in Fig. 5. The period is $48 \mathrm{~s}$, the width of the transition layer is 0.05 and the density ratio, $\rho_{\mathrm{i}} / \rho_{\mathrm{e}}=10$.

linear damping rates when analysing observations of propagating kink waves.

There are several possible extensions to this work, such as including stratification, field line curvature and magnetic field expansion. The density decrease in the direction of wave propagation is known to modify the amplitude of the velocity and magnetic field perturbations, increasing one and decreasing the other so that, when the wavelength is less than the stratification length, the perturbed Poynting flux remains constant. Most recently Soler et al. (2011b) have investigated the effect of stratification. When the density is decreasing away from the location of the driver, there is a competition between the increase in amplitude, due to the stratification, and a decrease due to the damping. Thus, we expect the same competition to occur between the Gaussian damping and the amplitude increase. If the density varies over a distance much shorter than the wavelength, there may be reflection.

The effect of field line curvature on the kink wave has been reviewed recently by Van Doorsselaere et al. (2009). For a semitoroidal loop, the curved extension to the straight cylinder considered here, they find that curvature does not change either the normal mode frequency or the damping due to the narrow inhomogeneous layer. Only when $\epsilon$, the ratio of the transition layer width to loop radius, is large is there a significant effect. However, large values of $\epsilon$ cannot be accurately treated by the Thin Tube, Thin Boundary approximation used here. The equations for the evolution of propagating, damped kink modes for large values of $\epsilon$ must be solved numerically (see Pascoe et al. 2013, in this issue).

The expansion of the flux tube cross section has been studied by, for example, De Moortel et al. (2000) and Smith et al. (2007). As the flux tube widens, the wavelength shortens. This 
will enhance the damping through mode coupling. However, the shortening of the wavelength will eventually invalidate the long wavelength assumption of the thin tube. If the expansion of the magnetic field is characterised by the length $L_{\mathrm{A}}$, we would expect the Gaussian damping to be the dominate effect for $L_{\mathrm{A}}>L_{\mathrm{g}}$, where $L_{\mathrm{g}}$ is defined in Eq. (44). On the other hand, if $L_{\mathrm{A}}<L_{\mathrm{g}}$, the Gaussian damping envelope is likely to be modified.

The Gaussian form of the damping demonstrated in this paper may be modified by the various extensions to the straight, unstratified plasma cylinder described above. However, it is clear that the straightforward application of a simple linear exponential damping, while easy to apply, may give misleading results. A detailed comparison of these results when used for coronal seismology, is discussed in the accompanying paper by Pascoe et al. (2013).

Throughout the paper we have used the term "mode coupling" to describe the conversion of energy from the compressional (kink-like) driven wave to an incompressible (Alfvénlike) wave. Both terms refer to the same physical processes, but in different situations, and "mode coupling/conversion" can be thought of as more general than the term "resonant absorption". Consider the system of equations driven harmonically in time but studied with different boundary conditions. In this paper, the field lines are open and the axial wavenumber, $k$, is determined by the solution to these equations. If, on the other hand, the ends of the field lines are tied and it is the radial boundary that is driven, then the axial wavenumber becomes a discrete quantity. There is a large volume of papers considering this second case. The main features are that the global kink mode resonates at one particular radius, where its energy is absorbed, and its eigenfunction is singular here. The fact that the location of the singularity corresponds to a resonant matching of kink and natural Alfvén frequencies has led to this solution being termed "resonant" absorption. The solution we describe differs in that we do not have tied ends to our field lines, so there is no quantized $k$ and no natural Alfvén frequency. Moreover, our solution does not have any singularities: there is simply an accumulation of energy around a particular radius, but there is no singularity. The location of energy accumulation may be identified by the matching the kink and Alfvén phase speeds (Allan \& Wright 2000). Of course, if $k$ was determined by line tied boundary conditions, then matching phase speeds is equivalent to matching natural frequencies. Finally, we note that there is no singularity in the solutions if the finite length loop case is studied as an initial value problem and is not driven continually in a harmonic manner.

This paper has presented a detailed mathematical derivation of the damping of propagating kink waves. Amid all the mathematical expressions, there are only a few key results that are necessary for applying these results in practice. A simple flow diagram, shown in Fig. 7, identifies the important equations to use and the conditions under which they apply. Obviously the thin boundary assumption is essential in deriving the appropriate expressions from integrating across the transition layer. Hence, the results in this paper will provide useful results for $\epsilon \lesssim 0.2$. While the general damped kink mode is described by Eq. (27), the much simpler equation, Eq. (47), that was derived only assuming $\kappa \ll 1$, is applicable for all density contrasts. Equation (47) can be solved numerically but, if $\epsilon \leq 0.05$ then Eq. (48) provides a very good approximation to the damping envelope. Finally, if $0.05<\epsilon \lesssim 0.2$, a useful approximation can be derived on using $\tilde{\eta}_{3}$ in Eq. (54) for general $z$ and Eq. (55) for small values of $\kappa k z / 2$.

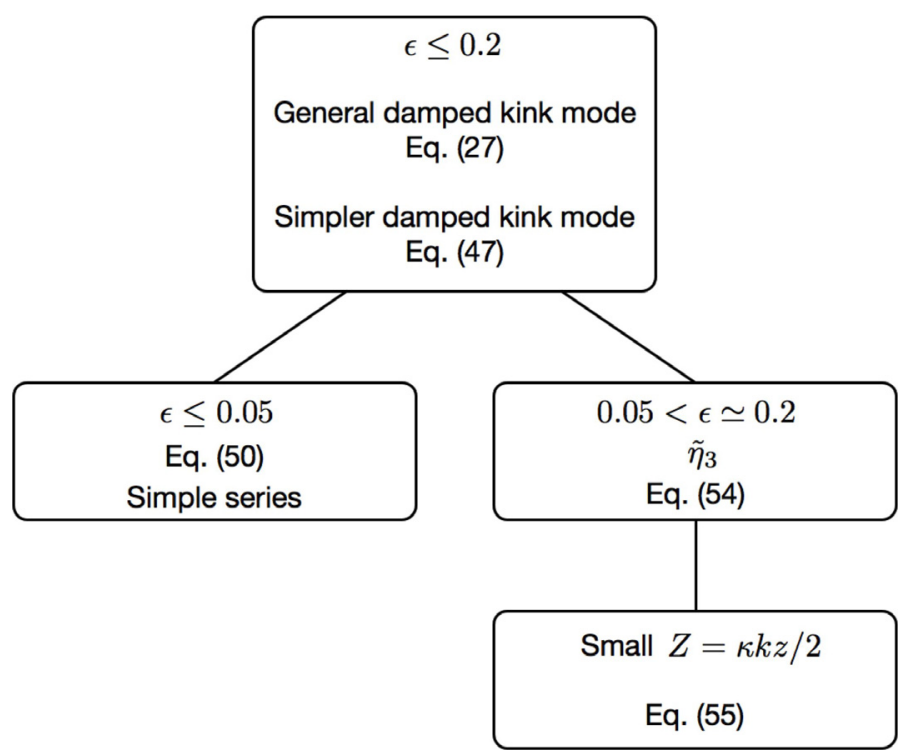

Fig. 7. Flow chart to identify the appropriate expressions to use.

Acknowledgements. D.J.P. acknowledges financial support from STFC. I.D.M. acknowledges support of a Royal Society University Research Fellowship. M.S.R. acknowledges the support by a Royal Society Leverhulme Trust Senior Research Fellowship and by an STFC grant. J.T. acknowledges support from the Spanish Ministerio de Educación y Ciencia through a Ramón y Cajal grant, financial support from MICINN/MINECO and FEDER Funds through grant AYA2011-22846 and funding from CAIB through "Grups Competitius" scheme and FEDER Funds is also acknowledged. The computational work for this paper was carried out on the joint STFC and SFC (SRIF) funded cluster at the University of St Andrews (Scotland, UK).

\section{References}

Abramowitz, M., \& Stegun, I. A. 1965, Handbook of Mathematical Functions, available at http://wWw.nr.com/aands/

Allan, W., \& Wright, A. N. 2000, JGR, 105, 317

Boyce, W. E., \& DiPrima, R. C. 2008, Elementary Differential Equations and Boundary Value Problems (John Wiley \& Sons)

Cirtain, J. W., Golub, L., Lundquist, L., et al. 2007, Science, 318, 1580

De Moortel, I., \& Nakariakov, V. M. 2012, Roy. Soc. Phil. Trans. A, 370, 3193

De Moortel, I., Hood, A. W., \& Arbert, T. D. 2000, A\&A, 354, 334

De Pontieu, B., McIntosh, S. W., Carlsson, M., et al. 2007, Science, 318, 1574

Dymova, M. V., \& Ruderman, M. S. 2006, A\&A, 457, 1059

Goossens, M., Hollweg, J. V., \& Sakurai, T. 1992, Sol. Phys., 138, 233

Goossens, M., Terradas, J., Andries, et al. 2012, A\&A, 503, A213

He, J.-S., Marsch, E., Tu, C.-Y., \& Tian, H. 2009, ApJ, 705, L217

Hollweg, J. V., \& Yang, G. 1988, J. Geophys. Res., 93, 5423

Ofman, L. 2010, Liv. Rev. Sol. Phys., 7, 4

Okamoto, T. J., Tsuneta, S., Berger, T. E., et al. 2007, Science, 318, 1577

Parnell, C. E., \& De Moortel, I. 2012, Roy. Soc. Phil. Trans. A, 370, 3217

Pascoe, D. J., Wright, A. N., \& De Moortel, I. 2010, ApJ, 711, 990

Pascoe, D. J., Wright, A. N., \& De Moortel, I. 2011, ApJ, 731, 73

Pascoe, D. J., Hood, A. W., De Moortel, I., \& Wright, A. N. 2012, A\&A, 539, A37

Pascoe, D. J., Hood, A. W., De Moortel, I., \& Wright, A. N. 2013, A\&A, 551, A40

Ruderman, M. S. 2011, A\&A, 534, A78 (Paper I)

Ruderman, M. S., \& Roberts, B. 2002, ApJ, 577, 475

Ruderman, M. S., \& Terradas, J. 2012, A\&A, submitted

Smith, P. D., Tsiklauri, D., \& Ruderman, M. S. 2007, A\&A, 475, 1111

Soler, R., Terradas, J., \& Goossens, M. 2011a, ApJ, 736, 10

Soler, R., Terradas, J., Verth, G., \& Goossens, M. 2011b, ApJ, 734, 80

Terradas, J., Goossens, M., \& Verth, G. 2010, A\&A, 524, A23

Tomczyk, S., \& McIntosh, S. W. 2009, ApJ, 697, 1384

Tomczyk, S., McIntosh, S. W., Keil, S. L., et al. 2007, Science, 317, 1192

Van Doorsselaere, T., Verwichte, E., \& Terradas, J. 2009, Space Sci. Rev., 149, 299

Verth, G., Terradas, J., \& Goossens, M. 2010, ApJ, 718, L102 
A. W. Hood et al.: Damping of kink waves by mode coupling. I.

\section{Appendix A: Derivation of kink mode equation}

We give a short derivation of the basic equations, starting from Eqs. (3)-(5). The solutions are obtained in the three regions, inside the flux tube, outside the flux tube and in the transition layer.

\section{A.1. Internal solution}

Using the thin tube (or long wavelength) limit, the internal solutions can be expressed as

$\xi_{r}=\eta(z, t), \quad \xi_{\varphi}=\eta(z, t), \quad \frac{\mu_{0} P}{B_{0}^{2}}=r \mathcal{L}_{\mathrm{i}} \eta$

Hence, at $r=R-l / 2=R(1-\epsilon / 2)$, we have

$\xi_{r}=\eta, \quad \frac{\mu_{0} P_{\mathrm{i}}}{B_{0}^{2}}=R\left(1-\frac{\epsilon}{2}\right) \mathcal{L}_{\mathrm{i}} \eta$.

\section{A.2. External solution}

Again using the thin tube limit, we have

$\xi_{r}=\frac{R^{2}(1+\epsilon / 2)^{2}}{r^{2}} \xi_{\mathrm{e}}(z, t), \quad \xi_{\varphi}=-\xi_{r}, \quad \frac{\mu_{0} P}{B_{0}^{2}}=-\frac{R^{2}(1+\epsilon / 2)^{2}}{r} \mathcal{L}_{\mathrm{e}} \xi_{\mathrm{e}}(z, t)$.

Hence, at $r=R+l / 2=R(1+\epsilon / 2)$, we have

$\xi_{r}=\xi_{\mathrm{e}}, \quad \frac{\mu_{0} P_{\mathrm{e}}}{B_{0}^{2}}=-R\left(1+\frac{\epsilon}{2}\right) \mathcal{L}_{\mathrm{e}} \xi_{\mathrm{e}}$

Since both $\xi_{r}$ and $P$ are continuous across the transition layer as $\epsilon \rightarrow 0$, we can state $\xi_{\mathrm{e}}=\eta+\delta \xi_{r}, \quad P_{\mathrm{e}}=P_{\mathrm{i}}+\delta P$, where both $\delta \xi_{r}$ and $\delta P$ tend to zero as $\epsilon \rightarrow 0$. Using (A.4), we have, correct to $O\left(\epsilon^{2}\right)$,

$\begin{aligned} \xi_{\mathrm{e}} & =\eta+\delta \xi_{r} \\ -\mathcal{L}_{\mathrm{e}}\left(\eta+\delta \xi_{r}\right)\left(1+\frac{\epsilon}{2}\right) & =\mathcal{L}_{\mathrm{i}} \eta\left(1-\frac{\epsilon}{2}\right)+\frac{\mu_{0} \delta P}{R B_{0}^{2}}\end{aligned}$

Rearranging Eq. (A.5), the final equation for the propagating kink mode is

$\mathcal{L}_{\mathrm{k}} \eta=-\mathcal{M} \equiv-\frac{1}{2}\left(\mathcal{L}_{\mathrm{e}} \delta \xi_{r}+\frac{1}{R} \frac{\mu_{0} \delta P}{B_{0}^{2}}-\frac{\epsilon}{2} \mathcal{L}_{\mathrm{i}} \eta+\frac{\epsilon}{2} \mathcal{L}_{\mathrm{e}} \eta\right)$.

Note that the right hand side of (A.6) is of $O(\epsilon)$. It is the leading order expressions for $\delta \xi_{r}$ and $\delta P$ that we now need to calculate and this is done from the transition layer solutions.

\section{A.3. Transition layer solution}

Integrating Eq. (3) across the thin transition layer, we have

$$
\begin{aligned}
{\left[r \xi_{r}\right]_{R-l / 2}^{R+l / 2} } & =R(1+\epsilon / 2) \xi_{\mathrm{e}}-R(1-\epsilon / 2) \eta=\int_{R-l / 2}^{R+l / 2} \xi_{\varphi} \mathrm{d} r \\
\delta \xi_{r}+\epsilon \eta & =\frac{1}{R} \int_{R-l / 2}^{R+l / 2} \xi_{\varphi} \mathrm{d} r+O\left(\epsilon^{2}\right) .
\end{aligned}
$$

Integrating (4) we have

$\frac{\mu_{0} \delta P}{R B_{0}^{2}}=\frac{1}{R} \int_{R-l / 2}^{R+l / 2} \mathcal{L} \xi_{r} \mathrm{~d} r=\frac{1}{R} \int_{R-l / 2}^{R+l / 2} \mathcal{L} \eta \mathrm{d} r+O\left(\epsilon^{2}\right)$

Remembering that $\eta$ is independent of $r, \int_{R-l / 2}^{R+l / 2} \mathcal{L} \mathrm{d} r$ is $l$ times the average value of the operator $\mathcal{L}$ and, for the linear density profile, the average is $\mathcal{L}_{\mathrm{k}}$, thus,

$\frac{1}{R} \int_{R-l / 2}^{R+l / 2} \mathcal{L} \xi_{r} \mathrm{~d} r=\epsilon \mathcal{L}_{\mathrm{k}} \eta=O\left(\epsilon^{2}\right)$, 
since $\mathcal{L}_{\mathrm{k}} \eta=O(\epsilon)$. Hence, for the linear density profile

$\frac{\mu_{0} \delta P}{R B_{0}^{2}}=O\left(\epsilon^{2}\right)$

We substitute Eq. (A.7) into Eq. (A.6) and, using both Eq. (A.10), $\mathcal{L}_{\mathrm{i}}+\mathcal{L}_{\mathrm{e}}=2 \mathcal{L}_{\mathrm{k}}$ and that again $\mathcal{L}_{\mathrm{k}} \eta=O(\epsilon)$, this results in the propagating kink mode equation

$\mathcal{L}_{\mathrm{k}} \eta=-\frac{1}{2 R} \mathcal{L}_{\mathrm{e}} \int_{R-l / 2}^{R+l / 2} \xi_{\varphi} \mathrm{d} r$.

\section{A.4. Integration of $\xi_{\varphi}$ across the transition layer}

In this section we evaluate

$\frac{1}{2 R} \int_{R-l / 2}^{R+l / 2} \xi_{\varphi} \mathrm{d} r=\frac{\epsilon}{4} \int_{-1}^{1} \xi_{\varphi} \mathrm{d} X$.

Using the solution for $\xi_{\varphi}$ given by (26), the integral across the transition layer is made up of four terms. These are evaluated in turn.

\section{A.4.1. Term 1}

Now the integral of the first term on the RHS of Eq. (26), due to the radial profile of the driving boundary condition of $\xi_{\varphi}$, is

$$
\begin{aligned}
& \int_{-1}^{1} a \operatorname{sgn}(-X) \mathrm{e}^{\mathrm{i}(\omega t-k \chi z)} \mathrm{d} X=a \int_{-1}^{0} \mathrm{e}^{\mathrm{i}(\omega t-k \sqrt{(1-\kappa X)})} \mathrm{d} X-a \int_{0}^{1} \mathrm{e}^{\mathrm{i}(\omega t-k \sqrt{(1-\kappa X)})} \mathrm{d} X \\
& =-\left(\frac{4 a}{k^{2} \kappa z^{2}}+\mathrm{i} \frac{4 a}{k \kappa z}\right) \mathrm{e}^{\mathrm{i} \omega T}+\frac{2 a}{k^{2} \kappa z^{2}}\left(\mathrm{e}^{\mathrm{i}(\omega t-k \sqrt{1-\kappa})}+\mathrm{e}^{\mathrm{i}(\omega t-k \sqrt{1+\kappa z)})}\right) \\
& +\mathrm{i} \frac{2 a}{k \kappa z}\left(\sqrt{1-\kappa} \mathrm{e}^{\mathrm{i}(\omega t-k \sqrt{1-\kappa})}+\sqrt{1+\kappa} \mathrm{e}^{\mathrm{i}(\omega t-k \sqrt{1+\kappa z})}\right) \\
& =-\mathrm{e}^{\mathrm{i} \omega T}\left[\frac{4 a}{k^{2} \kappa z^{2}}+\mathrm{i} \frac{4 a}{k \kappa z}-\frac{2 a}{k^{2} \kappa z^{2}}\left(\mathrm{e}^{\mathrm{i} k z(1-\sqrt{1-\kappa})}+\mathrm{e}^{\mathrm{i} k z(1-\sqrt{1+\kappa)})}\right)-\mathrm{i} \frac{2 a}{k \kappa z}\left(\sqrt{1-\kappa} \mathrm{e}^{\mathrm{i} k z(1-\sqrt{1-\kappa})}+\sqrt{1+\kappa} \mathrm{e}^{\mathrm{i} k z(1-\sqrt{1+\kappa)})}\right)\right] .
\end{aligned}
$$

For large $k z$, this is proportional to $(k z)^{-1}$. The influence of the choice of boundary condition does becomes less important after several wavelengths.

For small values of $k z$, we can expand the result in a series to show that the first term is

$\frac{2}{3 \kappa} a \mathrm{e}^{\mathrm{i} \omega T}\left(2-(1+\kappa)^{3 / 2}-(1-\kappa)^{3 / 2}\right) \mathrm{i} k z$.

For small $\kappa$, Term 1 can be expressed as

$-\frac{2 \mathrm{i} a}{Z}(1-\cos Z) a \mathrm{e}^{\mathrm{i} \omega T}+O(\kappa)$

where we have defined

$Z=\frac{\kappa s}{2}=\frac{\kappa k z}{2}$.

\section{A.4.2. Term 2}

The second term on the RHS integrates to give

$$
\begin{aligned}
& -\frac{a \kappa}{2} \int_{-1}^{1} \frac{\mathrm{e}^{\mathrm{i}(\omega t-k \sqrt{1-\kappa X} z)}}{\chi(1+\chi)} \mathrm{d} X=-\frac{a \kappa}{2} \mathrm{e}^{\mathrm{i} \omega T+\mathrm{i} 2 k z} \int_{-1}^{1} \frac{\mathrm{e}^{-\mathrm{i} k z(1+\sqrt{1-\kappa X})}}{\sqrt{1-\kappa X}(1+\sqrt{1-\kappa X})} \mathrm{d} X \\
& =-a \mathrm{e}^{\mathrm{i} \omega T+\mathrm{i} 2 k z}[\operatorname{Ci}(k z(1+\sqrt{1+\kappa}))-\operatorname{Ci}(k z(1+\sqrt{1-\kappa}))-\mathrm{iSi}(k z(1+\sqrt{1+\kappa}))+\mathrm{iSi}(k z(1+\sqrt{1-\kappa}))],
\end{aligned}
$$

where $\operatorname{Ci}(x)$ and $\operatorname{Si}(x)$ are the Cosine integral and Sine integral respectively, defined by

$\operatorname{Ci}(x)=\gamma+\ln x+\int_{0}^{x} \frac{1-\cos t}{t} \mathrm{~d} t$, 
A. W. Hood et al.: Damping of kink waves by mode coupling. I.

where $\gamma=0.57721 \ldots$ is Euler's constant and

$\operatorname{Si}(x)=\int_{0}^{x} \frac{\sin t}{t} \mathrm{~d} t$

Again this term is proportional to $(k z)^{-1}$ for large $k z$.

For small values of $k z$, it is easier to start from the integral expression. Hence, the first two terms in the Taylor series are

$a \mathrm{e}^{\mathrm{i} \omega T}\left\{-\ln \left[\frac{1+\sqrt{1+\kappa}}{1+\sqrt{1-\kappa}}\right]-2 \mathrm{i} k z \ln \left[\frac{1+\sqrt{1+\kappa}}{1+\sqrt{1-\kappa}}\right]+\mathrm{i} k z(\sqrt{1+\kappa}-\sqrt{1-\kappa})\right\}$.

For small values of $\kappa$, term 2 can be shown to reduce to

$-\frac{\kappa}{2} \frac{\sin Z}{Z} a \mathrm{e}^{\mathrm{i} \omega T}+O\left(\kappa^{2}\right)$

where $Z=\kappa k z / 2$, as above.

\section{A.4.3. Term 3}

The third term is

$$
\begin{aligned}
\int_{-1}^{1} \frac{\mathrm{i} k \kappa}{2 \chi} \mathrm{e}^{\mathrm{i}(\omega t-k \chi z)}\left\{\int_{0}^{z} \tilde{\eta}(u) \mathrm{e}^{-\mathrm{i} k(1-\chi) u} \mathrm{~d} u\right\} \mathrm{d} X & =\frac{\mathrm{i} k \kappa \mathrm{e}^{\mathrm{i} \omega T}}{2} \int_{0}^{z} \tilde{\eta}(u)\left\{\int_{-1}^{1} \frac{\mathrm{e}^{\mathrm{i} k(1-\sqrt{1-\kappa X})(z-u)}}{\sqrt{1-\kappa X}} \mathrm{~d} X\right\} \mathrm{d} u \\
& =\mathrm{e}^{\mathrm{i} \omega T} \int_{0}^{z} \tilde{\eta}(u) \frac{\mathrm{e}^{\mathrm{i} k(z-u)(1-\sqrt{1-\kappa})}-\mathrm{e}^{\mathrm{i} k(z-u)(1-\sqrt{1+\kappa)}}}{z-u} \mathrm{~d} u .
\end{aligned}
$$

The expansion of the coefficient of $\tilde{\eta}$ for small $k z$ gives to leading order

$\mathrm{i} k \mathrm{e}^{\mathrm{i} \omega T} \int_{0}^{k z} \tilde{\eta}(u)[\sqrt{1+\kappa}-\sqrt{1-\kappa}] \mathrm{d} u$.

The expansion for small $\kappa$ gives, where $Z=\kappa k z / 2$,

$2 \mathrm{i} \mathrm{e}^{\mathrm{i} \omega T} \int_{0}^{Z} \tilde{\eta}(u) \frac{\sin (Z-u)}{Z-u} \mathrm{~d} u+O(\kappa)$

\section{A.4.4. Term 4}

Consider the final term,

$$
\begin{aligned}
& \frac{\kappa}{2} \tilde{\eta}(z) \mathrm{e}^{\mathrm{i} \omega T} \int_{-1}^{1} \frac{1}{\chi(1+\chi)} \mathrm{d} X= \\
& \frac{\kappa}{2} \tilde{\eta}(z) \mathrm{e}^{\mathrm{i} \omega T} \int_{-1}^{1} \frac{1}{\sqrt{(1-\kappa X)}(1+\sqrt{(1-\kappa X)}} \mathrm{d} X, \\
& =-\tilde{\eta}(z) \mathrm{e}^{\mathrm{i} \omega T}\left[\ln (1+\sqrt{1-\kappa X)}]_{-1}^{1},\right. \\
& =\tilde{\eta}(z) \mathrm{e}^{\mathrm{i} \omega T} \ln \left[\frac{1+\sqrt{1+\kappa}}{1+\sqrt{1-\kappa}}\right] .
\end{aligned}
$$

The expansion for small $\kappa$ gives

$\mathrm{e}^{\mathrm{i} \omega T} \frac{\kappa}{2} \tilde{\eta}(z)+O\left(\kappa^{2}\right)$. 


\section{A.5. Final expression}

We can now bring together the expressions for all four terms to rewrite the kink mode equation, (A.11), as

$$
\begin{aligned}
& \mathcal{L}_{\mathrm{k}} \eta=-\frac{\epsilon \mathcal{L}_{\mathrm{e}}}{4} \int_{-1}^{1} \xi_{\varphi} \mathrm{d} X, \\
= & -\frac{\epsilon}{4} \mathcal{L}_{\mathrm{e}}\left\{-\mathrm{e}^{\mathrm{i} \omega T}\left[\frac{4 a}{k^{2} \kappa z^{2}}+\mathrm{i} \frac{4 a}{k \kappa z}-\frac{2 a}{k^{2} \kappa z^{2}}\left(\mathrm{e}^{\mathrm{i} k z(1-\sqrt{1-\kappa})}+\mathrm{e}^{\mathrm{i} k z(1-\sqrt{1+\kappa})}\right)\right.\right. \\
& \left.-\mathrm{i} \frac{2 a}{k \kappa z}\left(\sqrt{1-\kappa} \mathrm{e}^{\mathrm{i} k z(1-\sqrt{1-\kappa})}+\sqrt{1+\kappa} \mathrm{e}^{\mathrm{i} k z(1-\sqrt{1+\kappa})}\right)\right]+a \mathrm{e}^{\mathrm{i} \omega T} \mathrm{e}^{\mathrm{i} 2 k z}\left[\mathrm{Ci}\left(p_{4} k z\right)-\mathrm{Ci}\left(p_{3} k z\right)\right] \\
& \left.-\mathrm{i} a \mathrm{e}^{\mathrm{i} \omega T} \mathrm{e}^{\mathrm{i} 2 k z}\left[\operatorname{Si}\left(p_{4} k z\right)+\mathrm{i} \operatorname{Si}\left(p_{3} k z\right)\right]+\int_{0}^{z} \eta(u) \frac{\mathrm{e}^{\mathrm{i} k(z-u)(1-\sqrt{1-\kappa})}-\mathrm{e}^{\mathrm{i} k(z-u)(1-\sqrt{1+\kappa})}}{z-u} \mathrm{~d} u+\eta(z) \ln \left[\frac{p_{4}}{p_{3}}\right]\right\}, \\
= & -\frac{\epsilon \mathcal{L}_{\mathrm{e}}}{4}\left\{F(k z) \mathrm{e}^{\mathrm{i} \omega T}+\int_{0}^{z} \eta(k u) g(z-u) \mathrm{d} u+\eta \ln \left[\frac{p_{4}}{p_{3}}\right]\right\} \\
= & \mathrm{e}^{\mathrm{i} \omega T} \frac{\epsilon k^{2} \kappa}{4}\left(F(k z)+\int_{0}^{z} \tilde{\eta}(k u) g(z-u) \mathrm{d} u+\tilde{\eta} \ln \left[\frac{p_{4}}{p_{3}}\right]\right)-\frac{\epsilon}{4} \mathcal{L}_{\mathrm{k}}\left\{\mathrm{e}^{\mathrm{i} \omega T} F(k z)+\int_{0}^{z} \eta(k u) g(z-u) \mathrm{d} u+\eta \ln \left[\frac{p_{4}}{p_{3}}\right]\right\} .
\end{aligned}
$$

Expressing $\eta$ as $\tilde{\eta}(z) \mathrm{e}^{\mathrm{i} \omega T}$, our final equation is

$\mathcal{L}_{1} \tilde{\eta}=\frac{\epsilon k^{2} \kappa}{4}\left(F(k z)+\int_{0}^{z} \tilde{\eta}(k u) g(z-u) \mathrm{d} u+\tilde{\eta} \ln \left[\frac{p_{4}}{p_{3}}\right]\right)-\frac{\epsilon}{4} \mathcal{L}_{1}\left\{F(k z)+\int_{0}^{z} \tilde{\eta}(k u) g(z-u) \mathrm{d} u+\tilde{\eta} \ln \left[\frac{p_{4}}{p_{3}}\right]\right\}$,

where $p_{3}=1+\sqrt{1-\kappa}, p_{4}=1+\sqrt{1+\kappa}, \mathcal{L}_{1}=\mathrm{d}^{2} / \mathrm{d} z^{2}-2 \mathrm{i} k \mathrm{~d} / \mathrm{d} z$ and $\mathcal{L}_{\mathrm{e}}=-k^{2} \kappa+\mathcal{L}_{\mathrm{k}}$. In Eq. (A.14), the operator, $\mathcal{L}_{1}$, acting on the final terms in the curly brackets on the right hand side, results in terms that are small for $\kappa \ll 1$. In fact, the terms remain small even for $\kappa \leq 1 / 2$. Hence, we will neglect them and the comparison with the numerical results confirms this is a valid assumption (see Sect. 5).

Equation (A.14) is an inhomogeneous, integro-differential equation for $\tilde{\eta}(z)$, the slowly varying amplitude function that describes the damping of the kink mode. 